\section{(1) \\ CrossMark}

\title{
The changing landscape of chronic thromboembolic pulmonary hypertension management
}

\author{
Michael Madani ${ }^{1}$, Takeshi Ogo ${ }^{2}$ and Gérald Simonneau ${ }^{3,4,5}$
}

Affiliations: ${ }^{1}$ Division of Cardiovascular and Thoracic Surgery, University of California, San Diego, La Jolla, CA, USA. ${ }^{2}$ Division of Pulmonary Circulation, Dept of Advanced Medicine for Pulmonary Hypertension, National Cerebral and Cardiovascular Center, Suita, Osaka, Japan. ${ }^{3}$ Assistance Publique - Hôpitaux de Paris, Service de Pneumologie, Hôpital Bicêtre, Le Kremlin-Bicêtre, France. ${ }^{4}$ Université Paris-Sud, Laboratoire d'Excellence en Recherche sur le Médicament et Innovation Thérapeutique, Le Kremlin-Bicêtre, France. ${ }^{5}$ INSERM U-999, Le Kremlin-Bicêtre, France.

Correspondence: Michael Madani, Division of Cardiovascular and Thoracic Surgery, University of California, 9300 Campus Point Drive, San Diego, La Jolla 7892, CA 92037, USA. E-mail: mmadaniducsd.edu

@ERSpublications

Management of CTEPH is evolving rapidly, leading to improved patient outcomes http://ow.ly/rHrt30gUQWX

Cite this article as: Madani M, Ogo T, Simonneau G. The changing landscape of chronic thromboembolic pulmonary hypertension management. Eur Respir Rev 2017; 26: 170105 [https://doi.org/10.1183/ 16000617.0105-2017].

ABSTRACT For patients with chronic thromboembolic pulmonary hypertension (CTEPH), the current standard of care involves surgical removal of fibro-thrombotic obstructions by pulmonary endarterectomy. While this approach has excellent outcomes, significant proportions of patients are not eligible for surgery or suffer from persistent/recurrent pulmonary hypertension after the procedure. The availability of balloon pulmonary angioplasty and the approval of the first medical therapy for use in CTEPH have significantly improved the outlook for patients ineligible for pulmonary endarterectomy. In this comprehensive review, we discuss the latest developments in the rapidly evolving field of CTEPH. These include improvements in imaging modalities and advances in surgical and interventional techniques, which have broadened the range of patients who may benefit from such procedures. The efficacy and safety of targeted medical therapies in CTEPH patients are also discussed, particularly the encouraging data from the recent MERIT-1 trial, which demonstrated the beneficial impact of using macitentan to treat patients with inoperable $\mathrm{CTEPH}$, including those on background therapy. As the treatment options for CTEPH improve, hybrid management involving more than one intervention in the same patient may become a viable option in the near future.

\section{Introduction}

Chronic thromboembolic pulmonary hypertension (CTEPH) is a progressive pulmonary vascular disorder, classified as group 4 pulmonary hypertension (PH) in the European Society of Cardiology (ESC)/European Respiratory Society (ERS) guidelines $[1,2]$. The disease is usually a result of one or more episodes of pulmonary embolism and has been reported to occur in $0.6-4.4 \%$ of patients who have had a pulmonary

Received: Sept 122017 | Accepted after revision: Nov 252017

Support statement: Funding was received from Actelion Pharmaceuticals Ltd, Allschwil, Switzerland. Funding information for this article has been deposited with the Crossref Funder Registry.

Conflict of interest: Disclosures can be found alongside this article at err.ersjournals.com

Provenance: Publication of this peer-reviewed article was sponsored by Actelion Pharmaceuticals Ltd, Allschwil, Switzerland (principal sponsor, European Respiratory Review issue 146).

Copyright OERS 2017. ERR articles are open access and distributed under the terms of the Creative Commons Attribution Non-Commercial Licence 4.0. 
embolism [3-8]. CTEPH is characterised by macroscopic thromboembolic lesions within proximal or distal pulmonary arteries and microscopic pulmonary vasculopathy $[9,10]$. The clinical consequences of these pathological changes, which can include intimal thickening, vascular remodelling and plexiform lesions [10], are elevated pulmonary vascular pressures and increased pulmonary vascular resistance (PVR), which can lead to right heart failure and death $[9,10]$.

CTEPH patients, if left untreated, have a poor prognosis [11]: survival rates at 5 years have been reported at $30 \%$ for patients with mean pulmonary arterial pressure (mPAP) $>40 \mathrm{mmHg}$, and $10 \%$ for patients with an mPAP $>50 \mathrm{mmHg}$ [12]. However, a number of treatment options are now available and the management of this disease has advanced substantially in recent years. The standard of care in CTEPH is the surgical removal of thromboembolic material from the larger vessels using pulmonary endarterectomy (also known as pulmonary thromboendarterectomy). This provides major clinical and haemodynamic improvements and is associated with increased survival $[13,14]$. However, this procedure is invasive and can pose too high a risk to some patients. Furthermore, depending on the nature and location of the thrombi, and the extent of the underlying microvasculopathy, not all patients are candidates for surgery [14]. An emerging catheter-based interventional technique, called balloon pulmonary angioplasty (BPA) or percutaneous transluminal pulmonary angioplasty, is an alternative management option for some patients, who may, for example, have lesions too distal to be treated by pulmonary endarterectomy. CTEPH patients may also benefit from medical therapies that target the underlying microvascular disease.

Advances in treatment options are changing the landscape of CTEPH management and improvements in imaging modalities are enabling more accurate diagnoses and guiding treatment decisions in CTEPH patients [15]. This review discusses current and emerging treatment options for patients with CTEPH, and the potential for improving patient outcomes by utilising multiple treatment strategies.

\section{From diagnosis to initial management}

Recent evidence suggests that CTEPH is underdiagnosed due to a number of factors, including delayed referral and misdiagnosis $[15,16]$. Detailed guidance on diagnostic strategies is beyond the scope of this article and has been reviewed elsewhere $[1,2,15]$. Briefly, CTEPH is diagnosed when haemodynamic values indicate $\mathrm{PH}$, alongside mismatched perfusion defects (measured by ventilation/perfusion $\left(V^{\prime} / \mathrm{Q}^{\prime}\right)$ scanning) and indicators of CTEPH (observed using advanced imaging techniques) $[1,2] . V^{\prime} / Q^{\prime}$ scanning remains the gold-standard technique for diagnosing CTEPH $[1,2,12,15]$ and can exclude CTEPH with high sensitivity and specificity $(>90 \%)[1,2]$. Despite clear recommendations for its use in the ESC/ERS guidelines [1, 2], $V^{\prime} / Q^{\prime}$ scanning remains underutilised [17-19]. Modern imaging modalities such as computed tomography (CT) pulmonary angiography can provide useful clinical information in conjunction with $V^{\prime} / Q^{\prime}$ scanning, but cannot replace $V^{\prime} / Q^{\prime}$ scanning due to their lack of diagnostic specificity $[1,2,18]$. Once a diagnosis is confirmed, initial patient management consists of assessing operability for pulmonary endarterectomy (figure 1) and lifelong anticoagulation to prevent further thromboembolic events $[1,2]$.

With regard to disease-specific management, surgical, percutaneous interventional and/or pharmacological options are currently available to target proximal lesions, distal lesions and microvasculopathy (figure 2). Individual patients are likely to display more than one of these disease manifestations and therefore may benefit from more than one treatment approach. The options available for CTEPH management are the focus of the remainder of this article.

\section{Imaging of the pulmonary vasculature}

One essential step in patient management is the identification of the location and nature of thromboembolic lesions, as this determines the optimal therapy choice. Lesions in the proximal main, lobar and segmental arteries $[13,14]$ and, in some cases, distally located mid-segmental and sub-segmental branches [14], can be surgically removed by pulmonary endarterectomy. Distal lesions that are not deemed accessible to pulmonary endarterectomy may be amenable to $\operatorname{BPA}[21,22]$. It can be challenging to identify whether lesions are more amenable to pulmonary endarterectomy or BPA, and imaging beyond that performed as part of a diagnostic process is often required. As such, it is recommended that all patients are assessed and managed in expert centres by a multidisciplinary team $[1,2]$, including an experienced surgeon, CTEPH physicians, BPA experts and radiologists [13, 18].

Pulmonary angiography or selective pulmonary angiography is typically used for assessment of lesion morphology in CTEPH [15]. While digital subtraction pulmonary angiography is the current gold-standard method for characterising vessel morphology in CTEPH $[15,23]$, CT or magnetic resonance methodologies have recently grown in popularity due to their improved sensitivity and resolution [15]. CT pulmonary angiography is currently used to plan surgical procedures by revealing proximal vessels with thromboembolic obstructions $[15,24]$. This technique requires high levels of expertise and is used mostly 


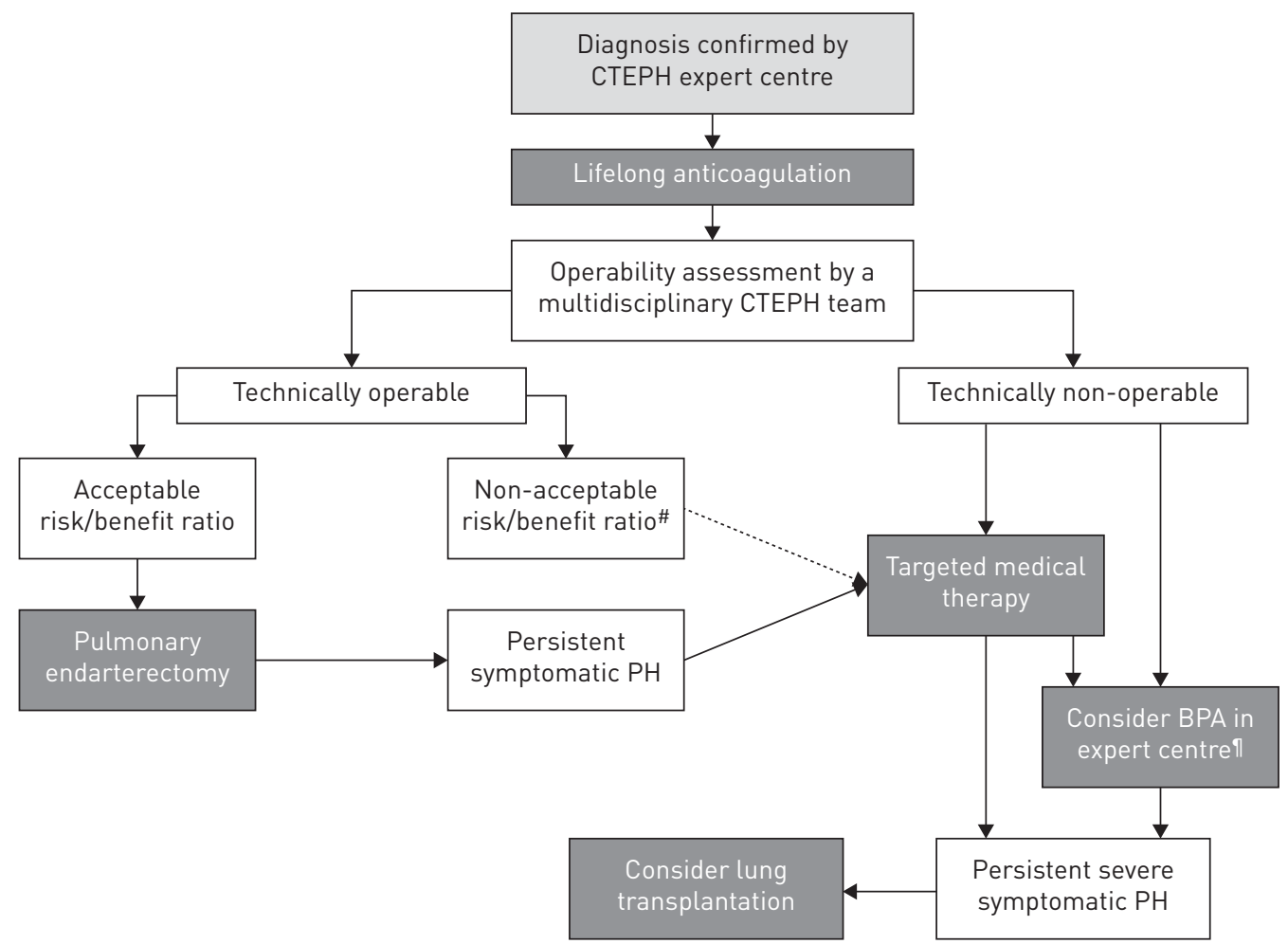

FIGURE 1 Treatment algorithm for managing chronic thromboembolic pulmonary hypertension (CTEPH), from the European Society of Cardiology/European Respiratory Society guidelines [1, 2]. Pulmonary endarterectomy is the standard of care for patients with technically operable CTEPH and an acceptable risk/benefit ratio for surgery. \#: technically operable patients with a non-acceptable risk/benefit ratio can be considered for balloon pulmonary angioplasty (BPA). ๆ: in some centres, medical therapy and BPA are initiated concurrently. $\mathrm{PH}$ : pulmonary hypertension. Reproduced and modified from [1, 2] with permission.

in experienced centres [15]. Recent studies have suggested that CT and magnetic resonance pulmonary angiography are superior to traditional pulmonary angiography methods in mapping lesions in proximal and segmental vessels $[15,24,25]$.

Advanced CT technologies including dual-energy CT, cone-beam CT (CBCT) and ECG-gated area detector $\mathrm{CT}(\mathrm{ADCT})$ are emerging noninvasive tools for evaluating changes to the vasculature in CTEPH $[15,22,24,26,27]$. Furthermore, both CBCT and ADCT have also been used successfully during BPA procedures, providing greater resolution than pulmonary angiography, particularly in small distal vessels [22]. Other imaging developments include the use of intravascular ultrasound to provide images of the interior vascular wall $[28,29]$. This technique allows a detailed assessment of lesion size in real time and therefore of the optimal balloon size required to treat a particular lesion [28].

In addition to assessing lesion type and location, therapeutic decision making should also consider patient-specific eligibility criteria and the relative merits and risks associated with each procedure.

\section{Pulmonary endarterectomy}

The ESC/ERS guidelines recommend pulmonary endarterectomy as the standard of care for patients with operable CTEPH and it should therefore be performed in all patients with accessible lesions who are suitable for surgery $[1,2]$. Overall, the proportion of patients who are deemed inoperable has been reported to range from $10 \%$ to $50 \%[30,31]$. However, based on our clinical experience, the number of patients that are considered inoperable may be over-estimated due to some patients being incorrectly diagnosed as having CTEPH.

The optimal surgical technique involves a full bilateral endarterectomy through the intima-media layer of the pulmonary arteries under deep hypothermia and circulatory arrest $[1,2]$. The deep hypothermia circulatory arrest method is considered optimal as it provides a clear operating field [18] and is associated with reproducible post-operative improvements [14], without impairments in brain function [32]. Unilateral endarterectomy is not advised as it has a number of disadvantages, including leaving untreated disease in the contralateral side [33]. 


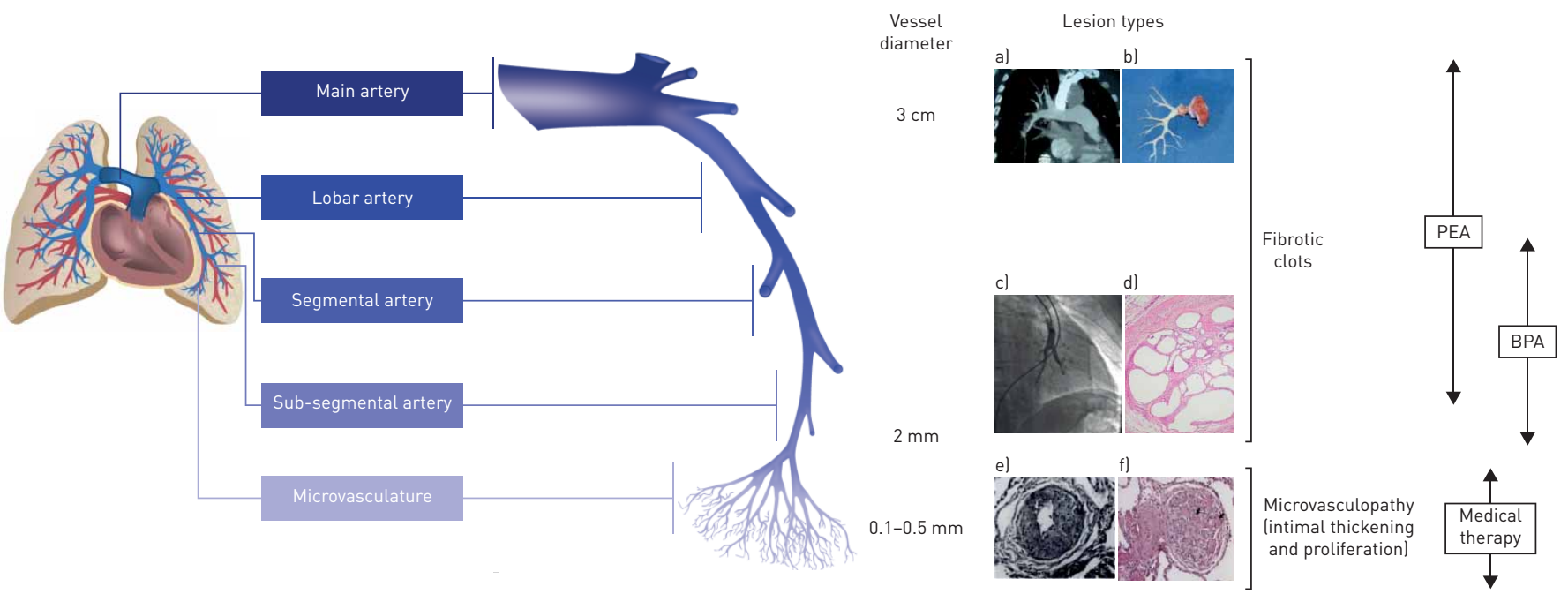

FIGURE 2 The management options for chronic thromboembolic pulmonary hypertension (CTEPH) target different pathogenic manifestations in different parts of the pulmonary vascular bed. A schematic representation of a pulmonary artery is shown (note that vessel diameter is not to scale). Pulmonary endarterectomy (PEA) is used to remove thromboembolic lesions primarily in the proximal main artery (diameter of $\sim 3 \mathrm{~cm}$ ), and lobar and segmental arteries [13, 14]; in expert surgical centres, lesions in distally located mid-segmental and sub-segmental branches can be targeted by PEA [14], down to vessels of $2 \mathrm{~mm}$ in diameter. Balloon pulmonary angioplasty (BPA) mainly targets distal lesions in the segmental and sub-segmental vasculature, down to small pulmonary arteries of $2-5 \mathrm{~mm}$ in diameter. Medical therapy targets microvasculopathy, including intimal thickening and fibromuscular proliferation, in vessels of $0.1-0.5 \mathrm{~mm}$ in diameter [10]. a) Computed tomography scan of a pulmonary artery. b) Organised fibrotic material removed during PEA. c) Selective pulmonary angiogram of segmental and sub-segmental pulmonary arteries, showing irregular vessel contour and occlusion, typical of CTEPH. d) Microscopic examination showing a luminal filling defect with recanalised chronic thrombus (web lesion) and no evidence of vasculopathy in the sub-segmental artery. e) Intimal fibromuscular proliferation. Reproduced from [20] with permission. f) Plexiform lesion and vessel occlusion due to vasculopathy and proliferation.

\section{Assessment of operability}

For patients with surgically accessible lesions (i.e. technically operable), pulmonary endarterectomy offers the best chance at improving long-term outcomes. However, it is associated with certain risks, including both the general risks associated with complex surgery and specific issues such as lung reperfusion injury, which occurs in $\sim 10 \%$ of patients [13]. Due to the risks involved, it is vital that a comprehensive assessment of the risk/benefit ratio for surgery is performed in all patients $[13,18]$.

Determining operability is complex $[1,2,18]$ and there are currently no standardised operability criteria [14]. General factors to consider when assessing suitability for surgery include age and general health [14], as well as comorbidities such as severe parenchymal disease, which can contraindicate surgery [34]. Measurement of a patient's haemodynamic status by right heart catheterisation is also an important part of risk assessment $[1,2]$. Although high pre-operative PVR $\left(>1000-1200 \mathrm{dyn} \cdot \mathrm{s} \cdot \mathrm{cm}^{-5}\right)$ is correlated with an increased risk of post-operative mortality $[13,14,35,36]$, patients with a high PVR are in a position to gain the most benefit from surgery, as they often show the greatest relative improvement in PVR after the procedure $[13,14]$. Therefore, high PVR should not necessarily be considered a contraindication for pulmonary endarterectomy $[14,37]$. High pre-operative PVR in conjunction with comparatively low levels of surgically accessible thrombotic material is indicative of significant microvascular disease [36, 38]. While this clinical scenario should be considered a risk factor for persistent or recurrent PH post-surgery, it is also not an absolute contraindication for pulmonary endarterectomy [39]. It is important to distinguish between patients who are technically inoperable and those who have accessible lesions, but are not suitable candidates for surgery for other reasons [14, 40,41], as the latter populations will require different management strategies. In order to guide therapeutic decisions, an updated surgical classification system for CTEPH has been developed [14]. The classification is based on the location of the disease (scored from 0 to IV; 0 for no disease, and I to IV from proximal (main) to distal (subsegmental)) and the subsequent degree of surgical difficulty [14].

Patients who are considered inoperable should be referred for a second opinion at another expert centre [18]. Operating on distal thromboembolic material is challenging and patients with lesions located in the distal segmental or sub-segmental arteries are more likely to be deemed technically inoperable than those with more proximal disease [14, 18, 41]. However, as some centres have reported successful pulmonary endarterectomy in patients with more distal disease [35], surgery should be considered in all patients [14]. 
Outcomes post-pulmonary endarterectomy

Pulmonary endarterectomy provides a number of clinical benefits. European registry data have shown that patients who underwent this procedure had improved 3-year survival rates compared with non-operated patients [42]. Furthermore, long-term data indicate high 10-year post-surgical survival rates (72\%) [43]. As well as having a positive impact on survival, improvements in World Health Organization (WHO) functional class (FC) have also been reported [44]. Additional benefits have been observed in a number of studies, including improvements in haemodynamics, particularly in PVR [35, 43, 45], and in quality of life $[32,46]$. Pulmonary endarterectomy is also able to reverse right ventricular remodelling, resulting in reduced right ventricular wall thickness and increased right and left ventricular ejection fractions and end-volumes [37].

Although there are a number of benefits associated with pulmonary endarterectomy, it remains a complex high-risk procedure. Despite this, the current in-hospital post-procedural mortality rate is low $(<5 \%[14,47])$ compared to two decades ago, due to refinements in surgical technique and increased experience in patient management [35]. It is notable that these improvements in outcome have been achieved in the setting of a more challenging surgical population, due to the increased numbers of patients with distal disease who are now considered operable [35].

Pulmonary endarterectomy is not always successful and up to $35 \%$ of patients have persistent $\mathrm{PH}$, defined as haemodynamic signs of $\mathrm{PH}$ remaining after surgery [48-52]. This is thought to result from incomplete removal of thrombi and/or the presence of small-vessel disease [13], and is a significant cause of post-operative morbidity and mortality [44]. CTEPH patients can also be affected by recurrent $\mathrm{PH}$ after pulmonary endarterectomy, which is the development of $\mathrm{PH}$ in patients who had previously displayed normal haemodynamics post-surgery. Recurrent $\mathrm{PH}$ is suspected to be the result of poor anticoagulation [14] or an ongoing distal vasculopathy [43]. Although recurrent $\mathrm{PH}$ is thought to be significantly less common than persistent $\mathrm{PH}[14,43]$, its prevalence may be underestimated. Most studies of recurrent $\mathrm{PH}$ only followed patients for up to 5 years; longer follow-up may have revealed a higher incidence of this complication as clinical experience suggests PH may recur as late as 10 years after surgery [43]. As such, patients should receive long-term haemodynamic follow-up after surgery in order to monitor for recurrent $\mathrm{PH}[43,49,53]$. A second pulmonary endarterectomy can be considered for patients with evidence of recurrent disease that is surgically accessible, or for those with persistent $\mathrm{PH}$ after surgery who may not have had a complete endarterectomy; however, these patients should be carefully selected by an expert team. Patients who would benefit the most are those with prior incomplete endarterectomy and/or embolectomy, without a true distal endarterectomy.

\section{Balloon pulmonary angioplasty}

For patients who are technically inoperable or who have been judged to have an unacceptable risk/benefit ratio for pulmonary endarterectomy, BPA and medical therapy are treatment options $[1,2]$. These treatment modalities can also be considered if persistent or recurrent $\mathrm{PH}$ occurs after surgery $[1,2]$. Although BPA is a relatively new technique [54], it is used with increasing frequency in some specialist centres. With the field of BPA rapidly evolving, it is expected that this trend will continue.

BPA is significantly less invasive than pulmonary endarterectomy; it does not require general anaesthesia or circulatory arrest and can be performed via a small surgical incision [22, 55]. During BPA, a guide wire inserted via the femoral or jugular vein [56] is used to accurately place a balloon at the site of a lesion or occlusion [55], with the assistance of imaging techniques such as selective pulmonary angiography, intravascular modalities, pressure wires or advanced CT $[22,56]$. Inflation of the balloon widens stenotic lesions and breaks intraluminal webs and bands in the distal pulmonary vasculature and restores vascular blood flow [56]. The lobe with the worst perfusion is often targeted first and more than one lobe may be treated in a single session in patients with permissive haemodynamics (mPAP $<40 \mathrm{mmHg}$ ) [55, 56]. Depending on the number and severity of the lesions, patients generally require multiple BPA sessions to adequately manage their disease [56].

\section{Assessment of suitability for BPA}

Currently there is no guidance or standardisation regarding assessment of suitability for BPA and it is dependent on the expertise and judgement of each individual centre. If imaging has identified the presence of BPA-accessible lesions, then other eligibility criteria for the procedure should be assessed. Current clinical experience has identified a number of conditions that may contraindicate BPA [21, 55]. These include severe renal dysfunction or an allergic reaction to iodine-based contrast medium. For the latter, the use of gadolinium-based contrast agents may help to avoid allergic reactions [56]. 
Outcomes after BPA

BPA has been shown to improve symptoms, function and haemodynamic parameters in patients [21, 22, 56-60]. Results from one experienced centre have shown an average reduction in PVR of 57\% [22], as well as improvements in functional class, exercise capacity and brain natriuretic peptide levels, at 3 months post-BPA [21, 22]. These positive results were maintained 1 year after the intervention [22]. Similar findings from another centre have demonstrated a positive effect of BPA on clinical and haemodynamic parameters 6 months post-BPA [57]. Improvements in a number of measures of right heart function have also been observed following BPA intervention, suggesting that this procedure contributes to reversal of right ventricular remodelling [60]. Long-term survival after BPA has been reported as 85\% after a mean follow-up period of 51 months [59].

Despite the benefits associated with BPA, not all patients respond satisfactorily to the procedure [58]. In a recent study, up to $23 \%$ of patients had persistent $\mathrm{PH}$ [58]. Persistent $\mathrm{PH}$ does not appear to affect 1-year survival; however, it is associated with significant impairment in functional capacity and increased symptom severity compared with that reported in patients without persistent $\mathrm{PH}$ [58]. To our knowledge, there are no published data on the prevalence of recurrent $\mathrm{PH}$ following BPA intervention, although clinical experience suggests that this does occur.

In addition to persistent or recurrent $\mathrm{PH}$, there are a number of risks associated with the BPA procedure itself [56], including vessel perforation, reperfusion injury and oedema, and haemoptysis [21, 61]. However, in the two decades since its development, procedural refinements have reduced the risks associated with performing BPA. This has resulted in low 1-year post-BPA mortality rates [22, 62] (no deaths were reported in 385 procedures in one experienced centre [22]) and reduced reperfusion pulmonary oedema rates compared with historical cases $[21,22,54]$. The use of smaller profile balloons $(1.5-2 \mathrm{~mm})$, particularly in the initial sessions, has contributed significantly to these improvements in safety $[22,56]$.

In specialist centres, the level of experience in performing BPA has increased rapidly, leading to the identification of a number of factors that can be used to predict outcome and complication rate after BPA. For example, the location and morphology of thromboembolic lesions are determinants of the success and complication rate following BPA [63]. Higher success rates and fewer complications were observed with web lesions and ring-like stenoses compared with tortuous lesions [63]. Assessment of the right ventricle prior to BPA may also provide prognostic information, with a recent study indicating that the risk of persistent $\mathrm{PH}$ following BPA is related to right ventricular function prior to the procedure [64]. The haemodynamic severity of $\mathrm{PH}$ at baseline is also associated with an increased risk of post-BPA complications [59]; however, severe $\mathrm{PH}$ does not necessarily contraindicate BPA as studies have shown that BPA can be used successfully in patients with high mPAP (reviewed in [55]).

\section{Medical therapy}

In parallel to the progress made in interventional and surgical techniques in recent years, advances have also been made in the field of medical therapy to target microvascular disease in patients with inoperable CTEPH $[1,2,65]$. There is a rationale for using medical therapies that are typically used in pulmonary arterial hypertension $(\mathrm{PAH})$ to treat patients with inoperable CTEPH, based on observations that PAH and CTEPH share similar histopathological changes $[10,20]$. Currently, only one pharmacological therapy is licensed for the treatment of CTEPH, the soluble guanylate cyclase stimulator riociguat $[1,2,65,66]$. Riociguat is approved for adults with inoperable CTEPH or those who have persistent or recurrent CTEPH following surgical treatment $[65,66]$, and its use is recommended in the ESC/ERS guidelines $[1,2]$.

Other medical therapies, including endothelin receptor antagonists, phosphodiesterase type 5 inhibitors and therapies targeting the prostacyclin pathway, are not currently approved for use in CTEPH, but are commonly used in clinical practice, both in inoperable patients and those with recurrent or persistent $\mathrm{PH}$ after surgery [30, 42, 65, 67]. The existing randomised controlled trial (RCT) evidence supporting the efficacy of medical therapies in the management of patients with CTEPH is summarised in the following sections and in table 1. Small retrospective and open-label studies investigating the efficacy of medical therapy in CTEPH have also been performed and are reviewed in a recent publication [11].

\section{Therapies that target the nitric oxide pathway}

Nitric oxide is an endogenous vasodilator, which is found at reduced levels in CTEPH patients [11, 72]. Two classes of medical therapy that target the nitric oxide pathway have been studied in RCTs involving treatment-naïve patients with inoperable $\mathrm{CTEPH}$ and persistent/recurrent $\mathrm{PH}$ post-pulmonary endarterectomy: soluble guanylate cyclase stimulators and phosphodiesterase type 5 inhibitors. 
TABLE 1 Randomised controlled trial data on the use of medical therapies in patients with chronic thromboembolic pulmonary hypertension

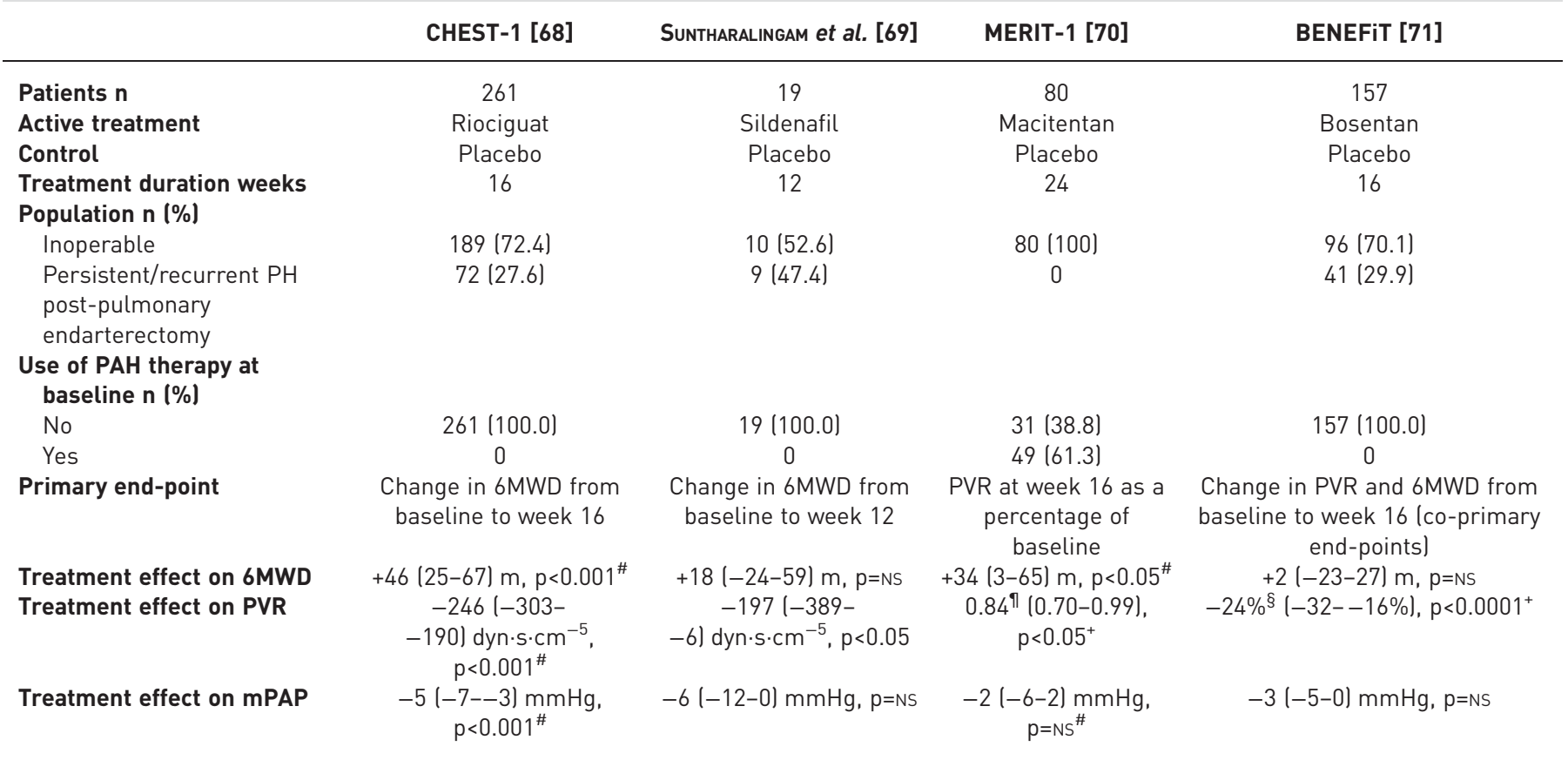

Data are presented as $\mathrm{n}, \mathrm{n}(\%)$ or mean difference $(95 \% \mathrm{CI})$, with $\mathrm{p}$-value, between active and control groups, unless otherwise stated. PH: pulmonary hypertension; PAH: pulmonary arterial hypertension; 6MWD: 6-min walk distance; PVR: pulmonary vascular resistance; mPAP: mean pulmonary arterial pressure; Ns: nonsignificant. "\#: treatment effect is the least-squares mean difference; ${ }^{\text {": }}$ corresponds to a $16 \%$ reduction, PVR decreased by $206 \mathrm{dyn} \cdot \mathrm{s} \cdot \mathrm{cm}^{-5}$ in the macitentan group and $86 \mathrm{dyn} \cdot \mathrm{s} \cdot \mathrm{cm}^{-5}$ in the placebo group; ${ }^{+}$: treatment effect is the ratio of geometric means; ${ }^{\S}$ : PVR decreased by $146 \mathrm{dyn} \cdot \mathrm{s} \cdot \mathrm{cm}^{-5}$ in the bosentan group and increased by $30 \mathrm{dyn} \cdot \mathrm{s} \cdot \mathrm{cm}{ }^{-5}$ in the placebo group.

Approval of the soluble guanylate cyclase stimulator riociguat for use in CTEPH was based on results from the CHEST-1 study [68]. In this trial, 16 weeks of treatment with riociguat led to improvements in the primary end-point of change from baseline in 6-min walk distance (6MWD) compared with placebo. In addition, improvements in PVR, N-terminal pro-brain natriuretic peptide levels and WHO FC (secondary outcomes) versus placebo were also reported $[68,73]$. During a 1-year open-label extension (CHEST-2), improvements in 6MWD and WHO FC were sustained, and the estimated 1-year survival rate was 97\% [74]. During the CHEST-1 study, greater rates of hypotension and haemoptysis were observed in the riociguat arm compared with the placebo arm [68].

The phosphodiesterase type 5 inhibitor sildenafil has also been investigated in an RCT in patients with CTEPH [69]. In this trial, no significant improvements were observed in the primary outcome of change in 6MWD after 12 weeks of sildenafil treatment compared with placebo, although significant improvements were reported for the secondary outcomes of WHO FC and PVR [69]. One serious adverse event of urticarial rash was observed during the study but sildenafil was otherwise well tolerated [69].

\section{Therapies that target the endothelin pathway}

Dysfunction of the endothelin pathway has been reported in patients with CTEPH [75], making it a promising target for pharmacological treatment. Endothelin receptor antagonists have been studied in two RCTs involving CTEPH patients. The most recent of these was the MERIT-1 study, which investigated the efficacy of macitentan in patients with inoperable CTEPH [70]. In this trial, macitentan significantly improved the primary outcome of change in PVR from baseline to week 16 compared with placebo [70]. This improvement in haemodynamics was accompanied by a significant and clinically meaningful improvement in the secondary outcome of change in 6MWD from baseline to week 24 [70]. Safety and tolerability were consistent with what had previously been observed for macitentan in the long-term SERAPHIN trial involving PAH patients [70, 76].

It is well established that patients with $\mathrm{PAH}$ benefit from treatment with a combination of therapies targeting multiple pathways. The MERIT-1 trial is the first RCT in CTEPH to allow PAH therapy at baseline, reflecting the real-world clinical scenario: $61 \%$ of patients were receiving treatment with another 
PAH therapy at baseline [70]. Of these, 96\% were receiving a phosphodiesterase type 5 inhibitor [70]. Subgroup analyses revealed that the beneficial treatment effects of macitentan on PVR and 6MWD in the subgroups of patients receiving and not receiving $\mathrm{PAH}$ therapy at baseline were consistent with those observed for the overall population [70].

The efficacy and safety of the endothelin receptor antagonist bosentan has also been assessed in an RCT in CTEPH (BENEFiT trial), in which patients with inoperable CTEPH or persistent/recurrent PH after pulmonary endarterectomy were enrolled [71]. Compared with placebo, bosentan significantly improved PVR, but not 6MWD, from baseline to week 16 (co-primary outcomes). There were also significant improvements in the secondary outcomes of total pulmonary resistance and cardiac index [71]. Bosentan was well tolerated and had a safety profile consistent with that observed in RCTs involving PAH patients [71].

\section{Therapies targeting the prostacyclin pathway}

Drugs targeting the prostacyclin pathway have been used to treat PAH for many years; however, the evidence for their efficacy in CTEPH is limited. There have been no RCTs of therapies targeting this pathway that have exclusively enrolled patients with CTEPH. However, a subgroup of 57 inoperable CTEPH patients was included in the placebo-controlled AIR study, which investigated the effect of inhaled iloprost administration in $203 \mathrm{PH}$ patients [77]. In the overall population, a significant improvement in the composite primary end-point (defined as an increase of $>10 \%$ in $6 \mathrm{MWD}$ from baseline and an improvement in WHO FC without disease worsening or death) was observed following 12 weeks of iloprost administration, compared with placebo [77]. However, no subgroup analyses were performed for the CTEPH group [77], making it difficult to determine the efficacy of inhaled iloprost in this particular population.

\section{Summary of medical management of CTEPH}

Several clinical trials have investigated medical therapies for the treatment of CTEPH, enrolling patients who have inoperable disease or persistent/recurrent $\mathrm{PH}$ after pulmonary endarterectomy [68-71]; the evidence from these trials indicates that medical therapy plays an important role in the management of such patients. Medical therapies are used with increasing frequency in the management of CTEPH in both Europe and the USA [11, 51, 78], illustrating the high medical need for management options beyond pulmonary endarterectomy and BPA. It should be noted, however, that for patients who are deemed operable, medical therapy is not a substitute for surgery $[1,2]$, and pulmonary endarterectomy remains the standard of care for patients with operable CTEPH $[1,2]$. Registry data indicate that medical therapies are being administered as both monotherapy and combination therapy in clinical practice [30]. Although combination therapy has been used in PAH for many years, and forms the standard of care for managing this progressive disorder, until recently there were no RCT data available to support this approach in CTEPH. The MERIT-1 study has now provided results to suggest that combining medical therapies could be effective in the management of CTEPH [70].

\section{Medical therapies as a bridge to pulmonary endarterectomy or BPA}

Medical therapies are also used in the clinic as a bridge to pulmonary endarterectomy or BPA [56, 78]. Studies have shown that administration of medical therapies can significantly reduce PVR in patients with severe CTEPH prior to pulmonary endarterectomy $[79,80]$, which may potentially improve their post-surgical prospects. However, it is not known whether medical therapy affects the integrity of the clot or the vessel wall and if this may increase the difficulty of subsequent endarterectomy. Medical therapy has also been used in small studies to stabilise patients' haemodynamics prior to undergoing BPA [29, 81]. Data on the use of bridging therapy in CTEPH patients are currently limited and prospective RCTs are needed to support this approach $[1,2]$.

\section{Future perspectives}

There have been many developments in the field of CTEPH in recent years, and this progress is expected to continue in the future. With multiple different management strategies available, the current treatment paradigm is likely to evolve to include a hybrid approach to management. Such an approach would involve employing combinations of pulmonary endarterectomy, BPA and medical therapy to target proximal, distal and microvascular disease in the same patient. While initial reports have demonstrated that this approach has potential [82-84], further prospective studies are warranted to determine the role that hybrid strategies can play in the future management of CTEPH.

Another area of CTEPH management that will require further study relates to overlap in the anatomical indication of pulmonary endarterectomy and BPA, as illustrated in figure 2. As experience with pulmonary endarterectomy and BPA grows, prospective randomised studies may be needed to compare the relative 
benefits and risks of pulmonary endarterectomy and BPA for lesions that could be targeted by both techniques. However, it may be difficult to perform such studies at present as BPA is still an emerging technique and there is no established protocol for its use. Furthermore, the success rate of pulmonary endarterectomy and BPA is dependent on the experience and technique of the operator, which would be difficult to account for in a clinical trial setting.

Currently, only one medical therapy (riociguat) is approved for the treatment of CTEPH. The recently completed MERIT-1 study for macitentan [70], and future trials, may lead to other drugs being approved to treat CTEPH, broadening the options available for the medical management of this disease. As the number of pharmacological options increases, the prospect of combining different agents to slow the progressive nature of CTEPH becomes increasingly attractive. This approach is likely to gain increasing importance in clinical practice in the coming years.

The potential role of medical therapy as a bridge to pulmonary endarterectomy or BPA also warrants additional study. The ongoing randomised RACE study (ClinicalTrials.gov identifier NCT02634203) will shed further light on this topic and provide additional information on the respective roles of BPA and medical therapy in the management of inoperable CTEPH patients. In the study, patients will initially receive either riociguat or $\mathrm{BPA}$, but will be allowed to switch treatments if a satisfactory response to the original therapy is not observed after 6 months. The trial will therefore provide information about the potential benefits of using riociguat as a bridging therapy prior to BPA, or as a complementary therapy following BPA.

\section{Conclusions}

The landscape of CTEPH management is evolving at a rapid rate. Recent increases in the number of available management options and refinements in surgical and interventional techniques have strengthened our capabilities to treat CTEPH. It is essential that appropriate management strategies are used for each patient and this requires careful evaluation in an expert centre by a multidisciplinary team with access to advanced imaging techniques. As CTEPH is a multifaceted disease, it is possible that the future standard of care will expand from using a single therapeutic option to one which involves a combination of surgical, interventional and medical therapies to target different manifestations of the disease. Based on the current pace of change, exciting updates to the treatment algorithm for CTEPH are anticipated in the near future.

\section{Acknowledgements}

The authors would like to thank James Glasper (nspm Ltd, Meggen, Switzerland) for medical writing assistance, funded by Actelion Pharmaceuticals Ltd (Allschwil, Switzerland).

\section{References}

1 Galiè N, Humbert M, Vachiery JL, et al. 2015 ESC/ERS Guidelines for the diagnosis and treatment of pulmonary hypertension: The Joint Task Force for the Diagnosis and Treatment of Pulmonary Hypertension of the European Society of Cardiology (ESC) and the European Respiratory Society (ERS). Eur Respir J 2015; 46: 903-975.

2 Galiè N, Humbert M, Vachiery JL, et al. 2015 ESC/ERS Guidelines for the diagnosis and treatment of pulmonary hypertension: The Joint Task Force for the Diagnosis and Treatment of Pulmonary Hypertension of the European Society of Cardiology (ESC) and the European Respiratory Society (ERS). Eur Heart J 2016; 37: 67-119.

3 Pengo V, Lensing AW, Prins $\mathrm{MH}$, et al. Incidence of chronic thromboembolic pulmonary hypertension after pulmonary embolism. N Engl J Med 2004; 350: 2257-2264.

4 Becattini C, Agnelli G, Pesavento R, et al. Incidence of chronic thromboembolic pulmonary hypertension after a first episode of pulmonary embolism. Chest 2006; 130: 172-175.

5 Klok FA, van Kralingen KW, van Dijk AP, et al. Prospective cardiopulmonary screening program to detect chronic thromboembolic pulmonary hypertension in patients after acute pulmonary embolism. Haematologica 2010; 95 : 970-975.

6 Miniati M, Monti S, Bottai M, et al. Survival and restoration of pulmonary perfusion in a long-term follow-up of patients after acute pulmonary embolism. Medicine 2006; 85: 253-262.

7 Surie S, Gibson NS, Gerdes VE, et al. Active search for chronic thromboembolic pulmonary hypertension does not appear indicated after acute pulmonary embolism. Thromb Res 2010; 125: e202-e205.

8 Dentali F, Donadini M, Gianni M, et al. Incidence of chronic pulmonary hypertension in patients with previous pulmonary embolism. Thromb Res 2009; 124: 256-258.

9 Lang IM, Dorfmüller P, Vonk Noordegraaf A. The pathobiology of chronic thromboembolic pulmonary hypertension. Ann Am Thorac Soc 2016; 13: Suppl. 3, S215-S221.

10 Simonneau G, Torbicki A, Dorfmüller P, et al. The pathophysiology of chronic thromboembolic pulmonary hypertension. Eur Respir Rev 2017; 26: 160112.

11 Hoeper MM. Pharmacological therapy for patients with chronic thromboembolic pulmonary hypertension. Eur Respir Rev 2015; 24: 272-282.

12 Fedullo P, Kerr KM, Kim NH, et al. Chronic thromboembolic pulmonary hypertension. Am J Respir Crit Care Med 2011; 183: 1605-1613.

13 Jenkins D. Pulmonary endarterectomy: the potentially curative treatment for patients with chronic thromboembolic pulmonary hypertension. Eur Respir Rev 2015; 24: 263-271. 
14 Jenkins D, Madani M, Fadel E, et al. Pulmonary endarterectomy in the management of chronic thromboembolic pulmonary hypertension. Eur Respir Rev 2017; 26: 160111.

15 Gopalan D, Delcroix M, Held M. Diagnosis of chronic thromboembolic pulmonary hypertension. Eur Respir Rev 2017; 26: 160108.

16 Gall H, Hoeper MM, Richter MJ, et al. An epidemiological analysis of the burden of chronic thromboembolic pulmonary hypertension in the USA, Europe and Japan. Eur Respir Rev 2017; 26: 160121.

17 McLaughlin VV, Langer A, Tan M, et al. Contemporary trends in the diagnosis and management of pulmonary arterial hypertension: an initiative to close the care gap. Chest 2013; 143: 324-332.

18 Kim NH, Delcroix M, Jenkins DP. Chronic thromboembolic pulmonary hypertension. J Am Coll Cardiol 2013; 62: D92-D99.

19 Gall H, Preston IR, Hinzmann B, et al. An international physician survey of chronic thromboembolic pulmonary hypertension management. Pulm Circ 2016; 6: 472-482.

20 Moser KM, Bloor CM. Pulmonary vascular lesions occurring in patients with chronic major vessel thromboembolic pulmonary hypertension. Chest 1993; 103: 685-692.

21 Ogo T. Balloon pulmonary angioplasty for inoperable chronic thromboembolic pulmonary hypertension. Curr Opin Pulm Med 2015; 21: 425-431.

22 Ogo T, Fukuda T, Tsuji A, et al. Efficacy and safety of balloon pulmonary angioplasty for chronic thromboembolic pulmonary hypertension guided by cone-beam computed tomography and electrocardiogram-gated area detector computed tomography. Eur J Radiol 2017; 89: 270-276.

23 Lang IM, Plank C, Sadushi-Kolici R, et al. Imaging in pulmonary hypertension. JACC Cardiovasc Imaging 2010; 3: $1287-1295$.

24 Ley S, Ley-Zaporozhan J, Pitton MB, et al. Diagnostic performance of state-of-the-art imaging techniques for morphological assessment of vascular abnormalities in patients with chronic thromboembolic pulmonary hypertension (CTEPH). Eur Radiol 2012; 22: 607-616.

25 Dong C, Zhou M, Liu D, et al. Diagnostic accuracy of computed tomography for chronic thromboembolic pulmonary hypertension: a systematic review and meta-analysis. PLoS One 2015; 10: e0126985.

26 Sanda Y, Fukuda T, Sugiyama M, et al. Organized thrombus in pulmonary arteries in patients with CTEPH using ECG-gated contrast CT. Radiological Society of North America, 99th Scientific Assembly and Annual Meeting, 2013. Abstract LL-CHE4326.

27 Fukuda T, Ogo T, Nakanishi N, et al. Evaluation of organized thrombus in distal pulmonary arteries in patients with chronic thromboembolic pulmonary hypertension using cone-beam computed tomography. Jpn J Radiol 2016; 34: 423-431.

28 D'Armini AM. Diagnostic advances and opportunities in chronic thromboembolic pulmonary hypertension. Eur Respir Rev 2015; 24: 253-262.

29 Mizoguchi H, Ogawa A, Munemasa M, et al. Refined balloon pulmonary angioplasty for inoperable patients with chronic thromboembolic pulmonary hypertension. Circ Cardiovasc Interv 2012; 5: 748-755.

30 Pepke-Zaba J, Delcroix M, Lang I, et al. Chronic thromboembolic pulmonary hypertension (CTEPH): results from an international prospective registry. Circulation 2011; 124: 1973-1981.

31 Peacock A, Simonneau G, Rubin L. Controversies, uncertainties and future research on the treatment of chronic thromboembolic pulmonary hypertension. Proc Am Thorac Soc 2006; 3: 608-614.

32 Vuylsteke A, Sharples L, Charman G, et al. Circulatory arrest versus cerebral perfusion during pulmonary endarterectomy surgery (PEACOG): a randomised controlled trial. Lancet 2011; 378: 1379-1387.

33 Banks DA, Pretorius GV, Kerr KM, et al. Pulmonary endarterectomy: Part II. Operation, anesthetic management, and postoperative care. Semin Cardiothorac Vasc Anesth 2014; 18: 331-340.

34 Banks DA, Pretorius GV, Kerr KM, et al. Pulmonary endarterectomy: Part I. Pathophysiology, clinical manifestations, and diagnostic evaluation of chronic thromboembolic pulmonary hypertension. Semin Cardiothorac Vasc Anesth 2014; 18: 319-330.

35 Madani MM, Auger WR, Pretorius V, et al. Pulmonary endarterectomy: recent changes in a single institution's experience of more than 2,700 patients. Ann Thorac Surg 2012; 94: 97-103.

36 Tanabe N, Sugiura T, Jujo T, et al. Subpleural perfusion as a predictor for a poor surgical outcome in chronic thromboembolic pulmonary hypertension. Chest 2012; 141: 929-934.

37 D'Armini AM, Zanotti G, Ghio S, et al. Reverse right ventricular remodeling after pulmonary endarterectomy. J Thorac Cardiovasc Surg 2007; 133: 162-168.

38 Galiè N, Kim NH. Pulmonary microvascular disease in chronic thromboembolic pulmonary hypertension. Proc Am Thorac Soc 2006; 3: 571-576.

39 Madani M, Mayer E, Fadel E, et al. Pulmonary endarterectomy. patient selection, technical challenges, and outcomes. Ann Am Thorac Soc 2016; 13: Suppl. 3, S240-S247.

40 Kim NH, Mayer E. Chronic thromboembolic pulmonary hypertension: the evolving treatment landscape. Eur Respir Rev 2015; 24: 173-177.

41 Jenkins DP, Biederman A, D'Armini AM, et al. Operability assessment in CTEPH: lessons from the CHEST-1 study. I Thorac Cardiovasc Surg 2016; 152: 669-674.e3.

42 Delcroix M, Lang I, Pepke-Zaba J, et al. Long-term outcome of patients with chronic thromboembolic pulmonary hypertension: results from an international prospective registry. Circulation 2016; 133: 859-871.

43 Cannon JE, Su L, Kiely DG, et al. Dynamic risk stratification of patient long-term outcome after pulmonary endarterectomy: results from the United Kingdom National Cohort. Circulation 2016; 133: 1761-1771.

44 Mayer E, Jenkins D, Lindner J, et al. Surgical management and outcome of patients with chronic thromboembolic pulmonary hypertension: results from an international prospective registry. J Thorac Cardiovasc Surg 2011; 141: 702-710.

45 Skoro-Sajer N, Marta G, Gerges C, et al. Surgical specimens, haemodynamics and long-term outcomes after pulmonary endarterectomy. Thorax 2014; 69: 116-122.

46 Archibald CJ, Auger WR, Fedullo PF, et al. Long-term outcome after pulmonary thromboendarterectomy. Am J Respir Crit Care Med 1999; 160: 523-528.

47 Hoeper MM, Madani MM, Nakanishi N, et al. Chronic thromboembolic pulmonary hypertension. Lancet Respir Med 2014; 2: 573-582. 
48 Freed DH, Thomson BM, Berman M, et al. Survival after pulmonary thromboendarterectomy: effect of residual pulmonary hypertension. J Thorac Cardiovasc Surg 2011; 141: 383-387.

49 Bonderman D, Skoro-Sajer N, Jakowitsch J, et al. Predictors of outcome in chronic thromboembolic pulmonary hypertension. Circulation 2007; 115: 2153-2158.

50 Ogino $\mathrm{H}$, Ando $\mathrm{M}$, Matsuda $\mathrm{H}$, et al. Japanese single-center experience of surgery for chronic thromboembolic pulmonary hypertension. Ann Thorac Surg 2006; 82: 630-636.

51 Condliffe R, Kiely DG, Gibbs JS, et al. Improved outcomes in medically and surgically treated chronic thromboembolic pulmonary hypertension. Am J Respir Crit Care Med 2008; 177: 1122-1127.

52 van der Plas MN, Surie S, Reesink HJ, et al. Longitudinal follow-up of six-minute walk distance after pulmonary endarterectomy. Ann Thorac Surg 2011; 91: 1094-1099.

53 Jamieson SW, Kapelanski DP, Sakakibara N, et al. Pulmonary endarterectomy: experience and lessons learned in 1,500 cases. Ann Thorac Surg 2003; 76: 1457-1462.

54 Feinstein JA, Goldhaber SZ, Lock JE, et al. Balloon pulmonary angioplasty for treatment of chronic thromboembolic pulmonary hypertension. Circulation 2001; 103: 10-13.

55 Ogawa A, Matsubara H. Balloon pulmonary angioplasty: a treatment option for inoperable patients with chronic thromboembolic pulmonary hypertension. Front Cardiovasc Med 2015; 2: 4.

56 Lang I, Meyer BC, Ogo T, et al. Balloon pulmonary angioplasty in chronic thromboembolic pulmonary hypertension. Eur Respir Rev 2017; 26: 160119.

57 Olsson KM, Wiedenroth CB, Kamp JC, et al. Balloon pulmonary angioplasty for inoperable patients with chronic thromboembolic pulmonary hypertension: the initial German experience. Eur Respir J 2017; 49: 1602409.

58 Tsuji A, Ogo T, Ueda J, et al. Predictors of residual pulmonary hypertension after balloon pulmonary angioplasty in patients with chronic thromboembolic pulmonary hypertension. Int J Cardiol 2017; 226: 118-120.

59 Andreassen AK, Ragnarsson A, Gude E, et al. Balloon pulmonary angioplasty in patients with inoperable chronic thromboembolic pulmonary hypertension. Heart 2013; 99: 1415-1420.

60 Broch K, Murbraech K, Ragnarsson A, et al. Echocardiographic evidence of right ventricular functional improvement after balloon pulmonary angioplasty in chronic thromboembolic pulmonary hypertension. $J$ Heart Lung Transplant 2016; 35: 80-86.

61 Inami T, Kataoka M, Shimura $\mathrm{N}$, et al. Incidence, avoidance, and management of pulmonary artery injuries in percutaneous transluminal pulmonary angioplasty. Int J Cardiol 2015; 201: 35-37.

62 Inami $T$, Kataoka $M$, Ando $M$, et al. A new era of therapeutic strategies for chronic thromboembolic pulmonary hypertension by two different interventional therapies; pulmonary endarterectomy and percutaneous transluminal pulmonary angioplasty. PLoS One 2014; 9: e94587.

63 Kawakami T, Ogawa A, Miyaji K, et al. Novel angiographic classification of each vascular lesion in chronic thromboembolic pulmonary hypertension based on selective angiogram and results of balloon pulmonary angioplasty. Circ Cardiovasc Interv 2016; 9: e003318.

64 Nagao M, Yamasaki Y, Abe K, et al. Energy efficiency and pulmonary artery flow after balloon pulmonary angioplasty for inoperable, chronic thromboembolic pulmonary hypertension: analysis by phase-contrast MRI. Eur J Radiol 2017; 87: 99-104.

65 Pepke-Zaba J, Ghofrani HA, Hoeper MM. Medical management of chronic thromboembolic pulmonary hypertension. Eur Respir Rev 2017; 26: 160107.

66 Bayer Pharma AG. Adempas (riociguat). Summary of Product Characteristics. www.ema.europa.eu/docs/en_GB/ document_library/EPAR_-_Product_Information/human/002737/WC500165034.pdf Date last updated: January 2017.

67 Robbins IM, Pugh ME, Hemnes AR. Update on chronic thromboembolic pulmonary hypertension. Trends Cardiovasc Med 2017; 27: 29-37.

68 Ghofrani HA, D'Armini AM, Grimminger F, et al. Riociguat for the treatment of chronic thromboembolic pulmonary hypertension. N Engl J Med 2013; 369: 319-329.

69 Suntharalingam J, Treacy CM, Doughty NJ, et al. Long-term use of sildenafil in inoperable chronic thromboembolic pulmonary hypertension. Chest 2008; 134: 229-236.

70 Ghofrani HA, Simonneau G, D'Armini AM, et al. Macitentan for the treatment of inoperable chronic thromboembolic pulmonary hypertension (MERIT-1): results from the multicentre, phase 2, randomised, double-blind, placebo-controlled study. Lancet Respir Med 2017; 5: 785-794.

71 Jaïs X, D'Armini AM, Jansa P, et al. Bosentan for treatment of inoperable chronic thromboembolic pulmonary hypertension: BENEFiT (Bosentan Effects in iNopErable Forms of chronIc Thromboembolic pulmonary hypertension), a randomized, placebo-controlled trial. J Am Coll Cardiol 2008; 52: 2127-2134.

72 Stasch JP, Evgenov OV. Soluble guanylate cyclase stimulators in pulmonary hypertension. Handb Exp Pharmacol 2013; 218: 279-313.

73 Kim NH, D’Armini AM, Grimminger F, et al. Haemodynamic effects of riociguat in inoperable/recurrent chronic thromboembolic pulmonary hypertension. Heart 2017; 103: 599-606.

74 Simonneau G, D'Armini AM, Ghofrani HA, et al. Riociguat for the treatment of chronic thromboembolic pulmonary hypertension: a long-term extension study (CHEST-2). Eur Respir J 2015; 45: 1293-1302.

75 Reesink HJ, Meijer RC, Lutter R, et al. Hemodynamic and clinical correlates of endothelin-1 in chronic thromboembolic pulmonary hypertension. Circ J 2006; 70: 1058-1063.

76 Pulido T, Adzerikho I, Channick R, et al. Macitentan and morbidity and mortality in pulmonary arterial hypertension. N Engl J Med 2013; 369: 809-818.

77 Olschewski H, Simonneau G, Galiè N, et al. Inhaled iloprost for severe pulmonary hypertension. $N$ Engl J Med 2002; 347: 322-329.

78 Jensen KW, Kerr KM, Fedullo PF, et al. Pulmonary hypertensive medical therapy in chronic thromboembolic pulmonary hypertension before pulmonary thromboendarterectomy. Circulation 2009; 120: 1248-1254.

79 Nagaya N, Sasaki N, Ando M, et al. Prostacyclin therapy before pulmonary thromboendarterectomy in patients with chronic thromboembolic pulmonary hypertension. Chest 2003; 123: 338-343.

80 Bresser P, Fedullo PF, Auger WR, et al. Continuous intravenous epoprostenol for chronic thromboembolic pulmonary hypertension. Eur Respir J 2004; 23: 595-600. 
81 Sugimura K, Fukumoto Y, Satoh K, et al. Percutaneous transluminal pulmonary angioplasty markedly improves pulmonary hemodynamics and long-term prognosis in patients with chronic thromboembolic pulmonary hypertension. Circ J 2012; 76: 485-488.

82 Shimura N, Kataoka M, Inami T, et al. Additional percutaneous transluminal pulmonary angioplasty for residual or recurrent pulmonary hypertension after pulmonary endarterectomy. Int J Cardiol 2015; 183: 138-142.

83 Kopec G, Stepniewski J, Waligora M, et al. Staged treatment of central and peripheral lesions in chronic thromboembolic pulmonary hypertension. Pol Arch Med Wewn 2016; 126: 97-99.

84 Wiedenroth CB, Liebetrau C, Breithecker A, et al. Combined pulmonary endarterectomy and balloon pulmonary angioplasty in patients with chronic thromboembolic pulmonary hypertension. J Heart Lung Transplant 2016; 35: $591-596$. 